PHS Scientific House

\title{
International Journal of Pharma Research and Health Sciences
}

Available online at www.pharmahealthsciences.net

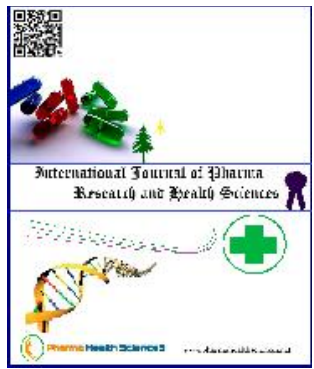

\section{Editorial}

\section{Side Effects of Baby Milk Powders: Awareness to the Lactating Mothers}

\author{
Harshita Mishra, Chitra Agarwal, Sisir Nandi * \\ Division of Pharmaceutical Chemistry, Global Institute of Pharmaceutical Education and Research, Affiliated to Uttarakhand \\ Technical University, Kashipur-244713, India.
}

ARTICLE INFO A B S T R A C T

Received: 16 Jun 2016

The present study governs that breast feeding is the superior for baby because it contains the necessity nutrients to maintain baby's immunity. A number of baby milk powder products are available in the market globally to meet the substitution of breast feeding. It was shown that milk powder preparations contain dissolved oxidized cholesterol which has been reported to produce cardiac problem. Oxidized cholesterol is called as oxysterols produced either by free radicals or by enzymes. Certain free radicals-derived oxysterols have been suspected of being initiators of atherosclerotic plaques. World Health organization (WHO) recommends exclusive breast feeding to the baby for at least 6 months. Breast milk gives infant steady supply of WBCs, antibodies, lymphocytes and vitamins, etc which are responsible for building immunity to the body of infants. According to WHO, early initiation of breast feeding within an hour immediately after birth could bring infant mortality rate down by $22 \%$. Therefore the present study is an awareness to the modern mothers of advanced society to save the life of their kids and future of the country by breast feeding and should not solely dependent on the baby milk powder formulations.

Keywords: Baby milk powder, oxysterols, atherosclerosis, no substitution for breast feeding.

Corresponding author *

Dr. Sisir Nandi

Associate Professor,

Division of Pharmaceutical Chemistry

Global Institute of Pharmaceutical Education and Research

(GIPER), Affiliated to Uttarakhand Technical University,

Kashipur-244713, Uttarakhand, India

E-mail:sisir.iicb@gmail.com

\section{INTRODUCTION}

Different branded baby milk powder products are formulated by various multinational companies to meet an alternative requirement of breast feeding ${ }^{1}$. The milk powder can be prepared by evaporating the milk to dryness usually by using spray drying. The purpose is to increase the shelf life of milk powders to preserve it for long duration and due to low moisture content they do not need to be refrigerated. Milk powders contain all the essential amino acids along with proteins, carbohydrates and some minerals such as calcium and potassium. According to USAID, average proportions (by weight) of major nutrients in non fat dry milk are $36 \%$ protein, $52 \%$ carbohydrates (predominantly lactose), calcium

IIIIIIII International Journal of Pharma Kesearch and Health Sciences. 
Int J Pharma Res Health Sci. 2016; 4 (4): 1249-1251

$1.3 \%$, and potassium $1.8 \%$. On the other hand, it contains an average of $25-27 \%$ protein, $36-38 \%$ carbohydrates, $26-40 \%$ fat, and $5-7 \%$ minerals. However, inappropriate storage conditions such as high relative humidity and high ambient temperature can significantly degrade the nutritive value of milk powder. As per WHO guidelines, an adult should take 400 to 500 milligrams of calcium a day to prevent bone fracture. Milk can fulfill nutritional supplements to the body and is ideal food for the mammals including human ${ }^{2}$. Apart from food and nutrition, dairy products such as dry whole milk, dry butter milk, dry whey products, non fat skimmed milk and dry dairy blends which are prepared from powdered milk are being commercially traded ${ }^{3}$. Too much use of milk powders to the baby is not good for health. An attempt has been made in the present study to aware to lactating mothers regarding side effects of feeding of the milk powders to the baby.

\section{POWDERS}

2. METHODS OF PREPARATION OF MILK Milk powders were originated as a modern method of preserving milk since long antiquity in the year of 1802. In 1832 the first commercial production of dried milk was initiated by $\mathrm{M}$ Dirchoff ${ }^{4}$.In 1837 and in 1855, William Newton and T.S. Grimwade patented a vacuum drying process and a procedure to prepare dried milk ${ }^{5}$. In modern times, milk powders were prepared by spray drying, drum drying and freeze drying processes respectively using pasteurized milk obtained from skimmed milk, whole milk, butter milk or whey. In spray drying, the milk is first concentrated in an evaporator and then the concentrated milk is sprayed into a heated chamber to make complete evaporation of the water content keeping finely divided powdered particles intact ${ }^{6}$.

Alternatively, the milk can be dried by drum drying where milk is applied as a thin film to the surface of a heated drum, and the dried milk solids are then scraped off. However, powdered milk made this way tends to have a cooked flavor, due to caramelization caused by greater heat exposure whereas in freeze drying, which preserves many nutrients in milk, compared to drum drying which alters the properties of the milk powder, such as its solubility in cold water, its flavor, and its bulk density. It was proved that on long storage of dried skim milk can deteriorate its quality and can hamper the health of baby ${ }^{7}$.

\section{RESULTS AND DISCUSSION}

The powdered milks are reported to contain oxysterols i.e. oxidized cholesterol in large amount than in fresh milk and oxidized cholesterol is hard to digest by the body. Oxysterols are the derivatives of cholesterol which are produced by either free radicals or enzymes. Having it regularly may cause illness, harmful effects of cholesterol like weight gain, high Blood Pressure, and in the worse cases it leads to many problems related to heart ${ }^{8}$.These are free radical cholesterols that are suspected to be the initiators of atherosclerotic plaques. In the past, powdered milk was not recommended for people with lactose tolerant. However, according to the United States Department of Agriculture, there is now low lactose content in powdered milk formula. The low lactose formula was originally developed in 1995 for military personnel who had requested to help for their soldiers that were lactose intolerant ${ }^{9-11}$. Therefore, babies should not be solely dependent on milk powders instead of breast feeding. As per WHO recommendation, breast feeding must be carried out for at least first six months and up to as much as of age for the proper growth and development of infants ${ }^{12}$. Baby can take colostrum through breast feeding which develops immunity to prevent the baby from jaundice attack and incorporates IgA by developing antibodies inside them which helps to fight against the foreign organisms. If a mother is unable to feed a baby, cow's milk can be used as a substituent. Breast feeding not only maintains nutrition, hydration and body temperature but also develops memory. These effects may not be known by the mothers. So the government should start awareness programmes for mother's to save the life of their child. Breast feeding decreases the risk of different life threatening diseases including respiratory tract infection, diarrhoea, asthma, celiac disease, Type-1 diabetes, leukemia and obesity in adulthood. It can trigger various biochemical reactions mediated by enzymes, hormones, growth factors, immunologic proteins and polyunsaturated fatty acids responsible for baby's normal growth intelligence and neuronal development ${ }^{13-14}$. Now-adays sometimes women are not ready to accept these issues seriously of feeding their babies either because of their busy schedule or sometimes in fear of loss of their beauty. Breast feeding can benefit to the mothers also through weight loss, less post-partum depression, decreased risk of breast cancer, CVS, rheumatoid arthritis and normalization of menstrual cycle ${ }^{15}$.

\section{CONCLUSION}

The present work is awareness to the lactating mothers having tendency to feed milk powder preparations in excess or before 6 months could be fatal for their baby of getting cardiac problem. They must have proper knowledge about the product what they are using. Due to presence of oxysterols in milk powders, it may lead to cause atherosclerosis which is lethal to the child. So lactating mothers should not be dependent on milk powders and it has been recommended for the breast feeding for a number of beneficial effects as given in the following Figure $1^{16}$.

\section{ACKNOWLEDGEMENTS}

Authors acknowledge Dr. S.C. Hazra, Professor \& Head, Dept. of Medicine, KPC Medical College and Hospital, Kolkata, India and sincere thanks to Dr. Benjamin $\mathrm{M}$. Nkowane, World Health Organization (WHO), Geneva, Switzerland for the constructive suggestions in the present work. Authors are sincerely thankful to GIPER, India. 


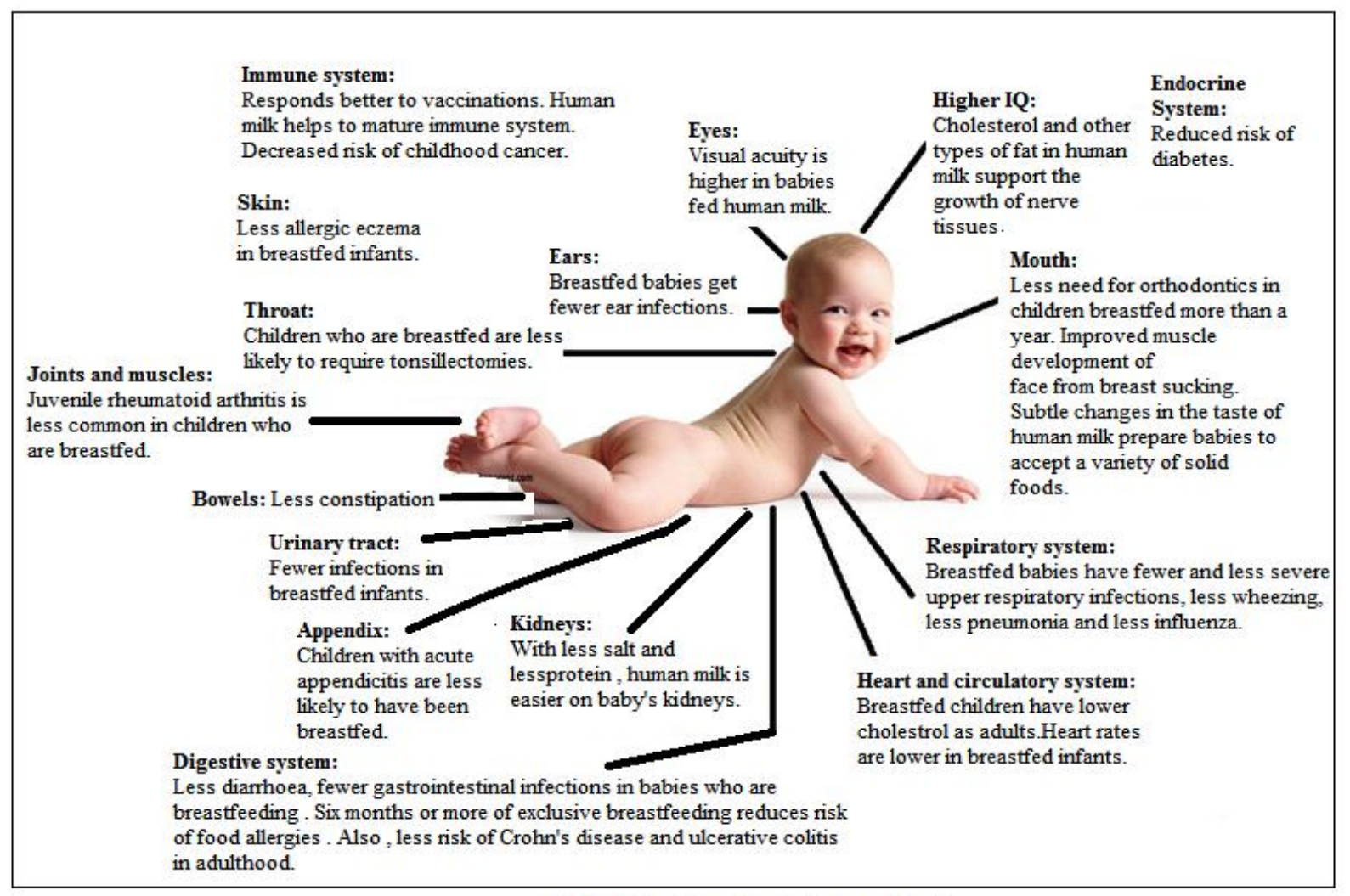

Fig 1: Baby is safe with breast feeding

\section{REFERENCES}

1. Vickers J. Powdered milk. Nature 1991;253: 103.

2. Okamoto M, Hayashi R. Chemical and Nutritional Changes of Milk Powder Proteins under Various Water Activities. Agric Biol Chem 1985; 49:1683-687.

3. FAO/WHO. Code of principles concerning milk and milk products (7th Ed.)Standard for whole milk powder, partly skimmed milk powder and skimmed milk powder. No. AS.FAO/WHO, 1973.

4. Blyth AW. Foods: Their Composition and Analysis: A Manual for the Use of Analytical Chemists and Others. C. Griffin, 1896. p 318.

5. Hunziker OF. Condensed milk and milk powder, $3^{\text {rd }}$ ed, 1920, p277.

6. Pearce KN. milk powder. Food science section, New Zealand dairy Research Institute. http://nzic.org.nz/ChemProcesses/dairy/

7. Henry KM, Kon SK, Lea CH, Smith JAB, White JCD. Deterioration on storage of dried skim milk. Nature 1946; 158: 348-349.

8. Hubbard RW, Ono Y, Sanchez A. Atherogenic effect of oxidized products of cholesterol, Progress in Food and Nutrition Science 1989;13:17-44.
9. Kadian A, Srivastava DN and Dabur RS. Evaluation of quality of milk powder manufactured in Hariana.Ind $\mathrm{J}$ Dairy Sci1988; 51: 285-288.

10. Fox PF, McSweeney P. Advanced Dairy Chemistry Lipids, volume2,Birkhauser, 2006, p 655.

11. Dabrowski WM, Sikorski ZE. Toxins in Food, CRC Press,2004: 296.

12. Benjamin RM. Public health in action: give mothers support for breastfeeding, Public Health Rep 2011; 126:622-623

13. Lawrence RA etal. Breast feeding and use of human milk, Paediatrics 2005, 115:496-506.

14. Victoria CG, Bahl R, Barrors AJG,Franca GVA,Horton S, Krasevec J, Murch S, Sankar MJ, Walker N, Rollins NC. Breast feeding in the $21^{\text {st }}$ century: epidemiology, mechanisms and lifelong effect. The Lancet 2016; 387: 475-90.

15. Galson SK. Mothers and children benefits from breastfeeding. J Am Diet Asso 2008; 108:1106.

16. http://robynhudson4309.blogspot.in/2013/08/breastfeedi ng.html 